\title{
Planning of Autonomous Multi-agent Intersection
}

\author{
Ilya I. Viksnin ${ }^{1, *}$ Igor A. Zikratov ${ }^{1}$, Andrei A. Shlykov ${ }^{1}$, Daria L. Belykh², Igor I. \\ Komarov and Gennady A. Botvin ${ }^{2}$ \\ ${ }^{1}$ ITMO University, Department of Secure Information Technology, Kronverkskiy pr., 49, Saint \\ Petersburg, Russia, 197101 \\ ${ }^{2} \mathrm{SPbU}$, Department of Information Systems in Economy, Universitetskaya emb., 7/9, Saint \\ Petersburg, Russia, 199034
}

\begin{abstract}
In this paper, we propose a traffic management system with agents acting on behalf autonomous vehicle at the crossroads. Alternatively to existing solutions based on usage of semiautonomous control systems with the control unit, proposed in this paper algorithm apply the principles of decentralized multi-agent control. Agents during their collaboration generate intersection plan and determinate the optimal order of road intersection for a given criterion based on the exchange of information about them and their environment. The paper contains optimization criteria for possible routes selection and experiments that perform in order to estimate the proposed model. Experiment results show that this model can significantly reduce traffic density compared to the traditional traffic management systems. Moreover, the proposed algorithm efficiency increases with road traffic density. Furthermore, the availability of control unit in the system significantly reduces the negative impact of possible failures and hacker attacks.
\end{abstract}

\section{Introduction}

One of the most significant current discussions in the automobile industry is about quickly developing technologies for robotic vehicle control. This is confirmed by the active participation of almost all the leading foreign automakers, many domestic and several research teams and companies in intelligent control system development in order to achieve driving without human control. Currently existing technical devices for vehicle control automation are considered as assistance to the driver in real road conditions when driver is dealing with individual management tasks. Such assistance systems contain a variety of Parking sensors and Parking Assistant, cruise control and autopilots, systems of emergency braking before the obstacle, etc. But current studies in this field are also aimed at the development of algorithms for automobile control for future when a person will not participate in the process of the unmanned vehicle control. [1]

It leads to developing new algorithms for autonomous vehicle control, which can handle a set of interrelated tasks[2-5]. These algorithms provide smooth car movement, correct

*Corresponding author: wixnin@cit.ifmo.ru 
route selection [6]. Also these algorithms make it possible to prevent collisions with obstacles [7] and other road users [8], organize safe and rapid intersection crossroad [913] and etc.

* Corresponding author: wixnin@cit.ifmo.ru

These algorithms are designed with two interrelated goals. The first one is increasing the efficiency of intersections. It can be achieved by using algorithms of optimal control during communication of autonomous vehicle network. The second one is providing road security. The security issues are resolved by eliminating human factor influence on the process of vehicle control and equipping drones and road infrastructure by the set of sensing devices, allowing real-time the traffic monitoring in various road and environmental conditions.

This paper organized as follows: Section 2 contains comparison of two different approaches for swarm robots control. In section 3 we present our algorithms, experiment and experiment results. Proposed algorithm allows to solve one of the typical challenges facing autonomous vehicle, particularly finding the optimal plan to safely crossing the intersection. In conclusion we formalized the results and directions for future study.

\section{Autonomous vehicle control models}

In this study, we consider a group of autonomous vehicles that come to intersection and /or locate at it as a multi-agent robotic system, characterized by the following factors:

- the unpredictable dynamics of the external environment;

- incomplete and contradictory knowledge of robots (agents) about conditions of the external environment and other road users;

- a variety of options for achieving goals, team structures, roles;

- the complexity of providing reliable communications in the distributed multiagent system.

The goal of developing system is to find a strategy, which allow to generate plan for crossroad intersection for each drone.

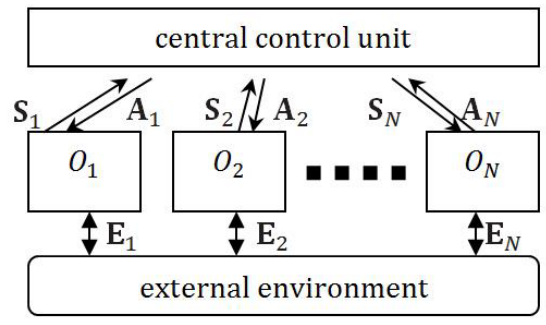

(1) centralized control model information exchange channel

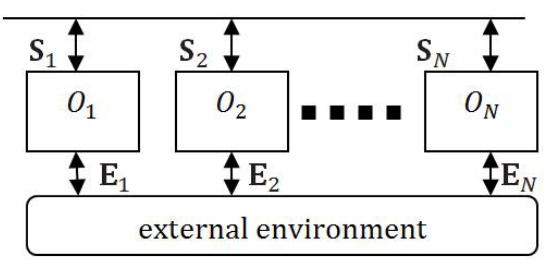

(2) decentralized control model

Fig. 1. Swarm robotics control models.

There are two fundamentally different approaches in swarm robotics for control the robots: (1) centralized control model and (2) decentralized control model. They are presented on Fig. 1.

Centralized control model includes central control unit and communication channels with all robots. In [9,10] proposed variety of algorithms for crossing the intersection by autonomous automobiles using a centralized management strategy. They are based on an idea that every intersection should be managed by an autonomous intelligent agent (central control unit). The central control unit or CCU controls each car individually, not a traffic 
light, which prevents or allows traffic on the entire road. According to one of this algorithms drone $O_{\mathrm{i}}$ comes to an intersection, connect to the CCU, send a message $S_{\mathrm{i}}$ to CCU. Central control unit simulates the situation taking into account other vehicles orders and incorporate $O_{\mathrm{i}}$ into the plan of intersection control due to previously received requests from the environment and other agents.

Significant disadvantages of this approach include: (1) the decision time increases exponentially depending on the number of objects $N$ in a group during usage of centralized group control strategy, and (2) the safety of intersection crossing strongly depends on the reliability of $\mathrm{CCU}$ and a communication channel with the agents, that forces to reserve critical communication channel facilities.

Decentralized control strategy bereft this drawback considerably making it an attractive to develop an algorithm for the solution using multi-agent communication and decisionmaking.

\section{The proposed model and its approbation}

Traffic management based on swarm intelligence involves the autonomous vehicles route selection which rely on traffic information received from other vehicles. The lack of a single center for decision-making complicates the route selection. It is necessary to define the fundamental principles of interaction and operation of autonomous vehicles to identify some criteria for plan selection and perform experiments in order to estimate the developing model performance.

\subsection{Principles of autonomous vehicles interaction}

For a complete understanding of the intersection management, we should describe intersection scheme. Schematic representation of investigated road intersection is presented in Fig 2. It contains an intersection of four roads, each may move in one direction only.

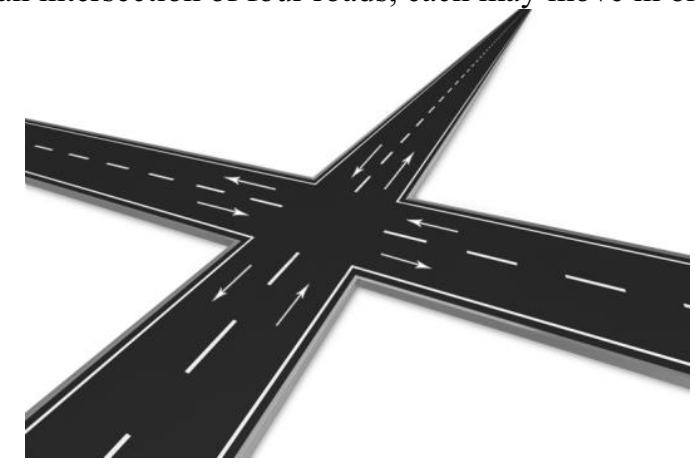

Fig. 2. Schematic representation of investigated road intersection.

During traffic from the start of road A to its end, autonomous automobiles, those who choose that route, will cross the intersection with road B. During the distributed intersection management vehicles collaborate at some time point. Let's the road consists of the set of elementary areas $\left\{p_{0}, \ldots, p_{\mathrm{n}}\right\}$. The crossroad is located on the area $P_{\mathrm{i}}, 0<i<n$. In this context $s$ represents a maximum speed on the road $A$ It is necessary to provide the interaction with other vehicles from the moment when the vehicle has enough distance for safe braking. Therefore, we need $s+1$ steps to stop. Hence, the full path of the braking is $(s(s+1)) / 2+1$ elementary areas. In an example that present on the Fig. 2 , the car must start the process of interaction with other vehicles at the moment when they located on position 
$p_{\mathrm{i}}((s(s+1)) / 2+1)$. For some simulation simplification, we make a decision that each car starts to communicate with others during that time, despite each car's speed.

Vehicle routing is based on the following basic principles:

1. Turning is performed according to scheme on Fig 3

2. Speed on the turning areas equals 1

3. Speed of moving vehicle on the previous or next time point may differ from the actual speed by more than 1

4. The vehicle tries moving with the maximum speed. At low speeds, it seeks the opportunity to restore it to maximum value

5. Speed more than maximum is unacceptable

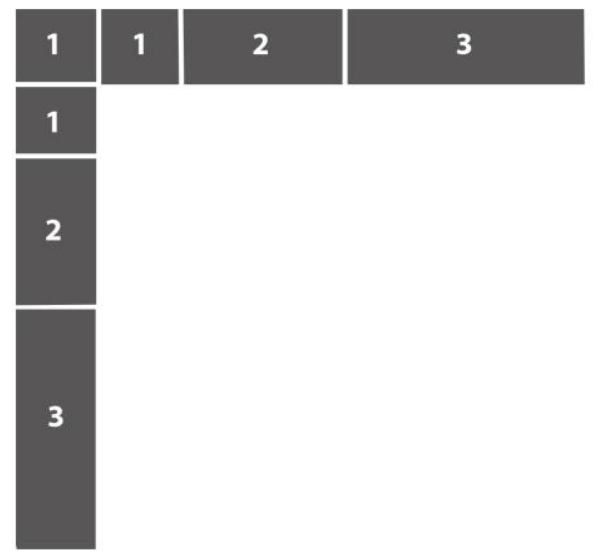

Fig. 3. Schematic representation of investigated road intersection.

Conflict is the point in time when two or more cars locate in the same elementary area. Rerouting proceed according to order of conflict situations occurrence. Each car involved in the conflict must change route using speed reduction. It allows to solve all other conflicts. In a general case, each conflict has two possible crossroad intersection plans. We use a variety of criteria to determine the best possible options.

According to Fig. 3, when the vehicle driving up to the crossroad elementary area after which it will have to change the motion direction, the vehicle must reduce speed to the minimum possible value. Speed reduction is carried out according to the general principles of smooth braking. After the vehicle passed the intersection, it accelerates smoothly to the maximum desired speed.

\subsection{Optimization criteria for crossroad intersection plans}

Exploring possible criteria of optimal route selection, we determined that the main criteria can be classified into three broad groups, according to the basic parameters of these criteria. In general case the data groups can be represented as follows:

1. Criteria based on collaborated vehicles speed characteristics

2. Criteria based on temporal traffic characteristics

3. Criteria based on the losses in distance due of car's speed reduction

Some concepts, which we will use further, should be clarified in order to consider each of the criteria groups. All vehicle characteristics involved in the process of specifying the routes can be divided into the desirable and real one. Desired characteristics are inherent to moving vehicle in the absence of other cars at the intersection. The actual characteristics are 
inherent to the vehicles movement after rerouting. Speed, time of location at the crossroad and some spatial characteristics can be both real and desired. Plan of movement should not be modified in the absence of conflict that means the actual characteristics are equal to desired.

It is necessary to enter the main used symbols before the definition of main optimization criteria:

Table 1. Used variables

\begin{tabular}{|c|c|}
\hline Variable & Description \\
\hline$v_{\mathrm{n}}{ }^{\text {act }}$ & The average actual speed of the $n$-th car \\
\hline$v_{\mathrm{n}}{ }^{\text {exp }}$ & The average expected speed of the $n$-th car \\
\hline$t_{\mathrm{n}}{ }^{\text {act }}$ & The actual intersection time of the $n$-th car \\
\hline$t_{\mathrm{n}}{ }^{\text {exp }}$ & The expected intersection time of the $n$-th car \\
\hline$d_{\mathrm{tn}}{ }^{\text {act }}$ & The actual covered distance of the $n$-th car at the moment $t$ \\
\hline$d_{\mathrm{tn}}{ }^{\exp }$ & The expected covered distance of the $n$-th car at the moment $t$ \\
\hline$N$ & Number of cars \\
\hline$T_{\mathrm{n}}$ & Intersection time of $n$-th car \\
\hline$D C_{\mathrm{n}}$ & Time without maximum speed of $n$-th car \\
\hline
\end{tabular}

The first criteria group is based on a driving cars speed during collaboration. In this article we use two following criteria from this group:

1. The average actual vehicle speed

2. The average difference between desired and actual speed

The criterion (1) aims to maximize the average speed, the criterion (2) intent to reduce the number of cars decelerations during collaboration. More precisely, the second criterion used for minimizing changes in the moving vehicle speed.

The second criteria group is aimed on optimization of temporal traffic characteristics. These group contain following main criteria:

3. Average actual time vehicles spent at the intersection

The usage of criterion (3) allows us to estimate how much time cars spent at the intersection in absolute numbers.

The third group of criteria is based on the distance losses because of the car did not pass because of forced reduction in speed:

4. Average loss in distance in terms of one unit of time

5. Average loss in distance due to the lower speeds

The criteria in this group are focused on possible reduction of costs from the speed decreasing. The criterion (4) allows us to calculate some costs for each delay, expressed in not traveled distance (the difference between the distance that could be overcome with maximum speed and distance that had been passed in reality), while the criterion (5) aims to minimize each car total losses.

\subsection{Performance indicators}

It is necessary to define some indicators in order to compare the model performance using each criterion.

Two performance systems are obvious: (1) average vehicle speed and (2) average time vehicle spent on the intersection. These indicators estimate the time needed to each vehicle for intersecting the crossroad. 


\subsection{Experiment results}

\subsubsection{A comparison of the classical and proposed model efficiency}

Three groups of experiments were conducted on the field, presented in Fig 2. The field is characterized with dimensions 10 at 10 . For a proper understanding of the proposed algorithm performance, it was decided to limit the roads to 4 lanes: two vertical (counter and passing) and two horizontal (counter and passing).

Groups of experiments differ in the probability of a vehicle appearance: $\mathrm{P}=1.0$ (there is at least one new car), $\mathrm{P}=0.5$ and $\mathrm{P}=0.2$. The number of appearing cars will be assigned randomly.

The experiments run 1000 times. The duration of each experiment is 1000 steps. Fig. 4 shows the results of the experiment.

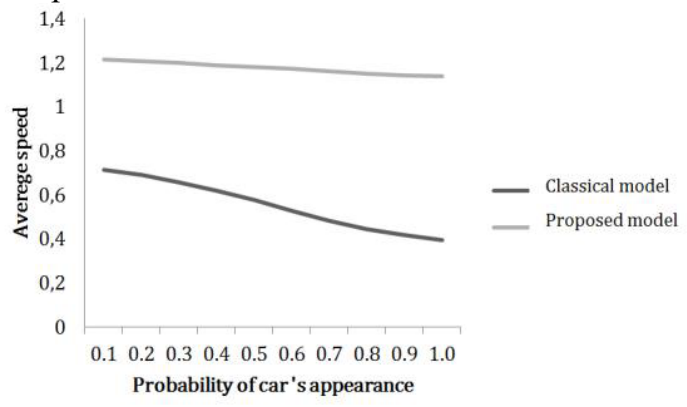

Fig. 4. The dependence of average speed from the probability of car appearance.

Above data represents that the algorithm helps to increase the speed of the crossroad intersection. It is noteworthy that the average traffic speed is decreasing by increasing the flux density in both cases, but this indicator is reduced by $15 \backslash \%$ less with the developed algorithm. Furthermore, average speed of autonomous vehicle is three times more, than the same one in classical model.

Average speed is a criterion for the proposed algorithm.

\subsubsection{A comparison of the efficiency criteria}

In order to compare criteria presented above we use field from Fig. 2. Based on developed model characteristics, experiment results are divided into two broad groups:

1. The probability of the car's appearance on the road less than 0.5 ;

2. The probability of the car's appearance on the road more than 0.5 .

This approach allows imagining the model capabilities under different conditions much better. This group choice is caused by the road capacity: in the first case less than half the road capacity is used and in the second case traffic load tends to reach maximum.

Table 2 presents the results of the model using different criteria for the first case, in Table 3 for the second one.

Table 2. A comparison of proposed model and same one that based on different criteria effectiveness (Probability $<0,5)$.

\begin{tabular}{|c|c|c|c|c|}
\hline Criteria & $\begin{array}{c}\text { Actual } \\
\text { Speed }\end{array}$ & $\begin{array}{c}\text { Expected } \\
\text { Speed }\end{array}$ & $\begin{array}{c}\text { Actual } \\
\text { Time }\end{array}$ & $\begin{array}{c}\text { Expected } \\
\text { Time }\end{array}$ \\
\hline 1 & 1,36 & 1,63 & 20,61 & 17,53 \\
\hline 2 & 1,36 & 1,63 & 20,59 & 17,53 \\
\hline
\end{tabular}




\begin{tabular}{|l|l|l|l|l|}
\hline 3 & 1,36 & 1,63 & 20,38 & 17,53 \\
\hline 4 & 1,33 & 1,63 & 20,90 & 17,53 \\
\hline 5 & 1,36 & 1,63 & 20,53 & 17,53 \\
\hline
\end{tabular}

Table 3. A comparison of proposed model and same one that based on different criteria effectiveness (Probability $>=0,5)$.

\begin{tabular}{|c|c|c|c|c|}
\hline Criteria & $\begin{array}{c}\text { Actual } \\
\text { Speed }\end{array}$ & $\begin{array}{c}\text { Expected } \\
\text { Speed }\end{array}$ & $\begin{array}{c}\text { Actual } \\
\text { Time }\end{array}$ & $\begin{array}{c}\text { Expected } \\
\text { Time }\end{array}$ \\
\hline 1 & 0,89 & 1,65 & 38,87 & 17,43 \\
\hline 2 & 0,90 & 1,65 & 38,08 & 17,39 \\
\hline 3 & 0,68 & 1,65 & 40,38 & 17,44 \\
\hline 4 & 0,81 & 1,65 & 41,95 & 17,44 \\
\hline 5 & 0,68 & 1,65 & 41,01 & 17,42 \\
\hline
\end{tabular}

As we can see from Table 2 and Table 3 different criteria should be used for different traffic options. It is much better to use criteria based on temporal road characteristics for cases with low congestion (it allows to reduce the actual time spent on the intersection). However, in case of a high workload, this criterion work slightly worse than any criterion, based on speed characteristics.

\section{Conclusion}

In this paper, we introduce a model for multi-agent car traffic planning, several optimization criteria for intersection plan, experiment performance in order to determine the efficiency of the proposed model with different criteria. The finding of the conducted experiments present, that the proposed model for intersection traffic management able to regulate the traffic more efficiently, due to increased autonomous vehicles average speed.

The results presented here may facilitate improvements in the further research taking into account the previously obtained results and new theoretical developments, regarding reduce of traffic congestion. Besides, we plan to evaluate the information security of the proposed model, analyse its resistance to destructive influences and develop protection methods from possible damage caused by the implementation of different threats.

\section{References}

1. Milanés V. et al. Clavileño. Evolution of an autonomous car. 13th Int. IEEE Conf. on ITSC, 1129-1134 (2010)

2. Varaiya P. Smart cars on smart roads: problems of control. IEEE Trans. on Autom. Cont. 38, 2 (1993)

3. Bazzan A.L.C. A Distributed Approach for Coordination of Traffic Signal Agents. Autonomous Agents and Multi-Agent Systems. 10, 1 (2005)

4. Beeson P. etc. Multi-agent Interactions in Urban Driving. J.of Phys.. 2, 1 (2008)

5. Hallé S., Chaib-draa B. A collaborative driving system based on multiagent modelling and simulations. Transp. Res. Part C: Emerging Technologies. 13, 4 (2005)

6. Carlino D., Boyles S. D., Stone P. Auction-based autonomous intersection management..16th Int.IEEE Conf.on ITSC, 529-534 (2013) 
7. Wuthishuwong C., Traechtler A.: Vehicle to infrastructure based safe trajectory planning for Autonomous Intersection Management. 13th Int. Conf. on ITST, 175-180 (2013)

8. Vahidi A., Eskandarian A. Research advances in intelligent collision avoidance and adaptive cruise control. IEEE Trans.on Intel. Transp. Syst. 4, 3 (2003)

9. Ho C., Reed N., Spence C. Multisensory in-car warning signals for collision avoidance. The Journal of the Human Factors and Ergonomics Society. 49, 6 (2007)

10. Au T., Zhang S., Stone P. Autonomous Intersection Management for SemiAutonomous Vehicles. Handbook of Transportation. 88-104 (2015)

11. Dresner K., Stone P. A multiagent approach to autonomous intersection management. Journal of artificial intelligence research. 591-656 (2008)

12. Wu J., Abbas-Turki A., El Moudni A. Cooperative driving: an ant colony system for autonomous intersection management. Appl. Int. 37, 2 (2012)

13. Zohdy I. H., Kamalanathsharma R. K., Rakha H. Intersection management for autonomous vehicles using icacc. 15th Int. IEEE Conf. on ITS, 1109-1114 (2012) 